\title{
L'informel internationalisé ou la subversion de la territorialité
}

\section{François Constantin}

\section{(2) OpenEdition \\ 1 Journals}

\section{Édition électronique}

URL : http://journals.openedition.org/conflits/245

DOI : $10.4000 /$ conflits. 245

ISSN : $1777-5345$

Éditeur :

CCLS - Centre d'études sur les conflits lilberté et sécurité, L'Harmattan

Édition imprimée

Date de publication : 15 mai 1996

ISSN : 1157-996X

Référence électronique

François Constantin, «L'informel internationalisé ou la subversion de la territorialité », Cultures \&

Conflits [En ligne], 21-22 I printemps-été 1996, mis en ligne le 15 mars 2006, consulté le 30 mars 2021.

URL : http://journals.openedition.org/conflits/245 ; DOI : https://doi.org/10.4000/conflits.245

Ce document a été généré automatiquement le 30 mars 2021.

Creative Commons License 


\title{
L'informel internationalisé ou la subversion de la territorialité
}

\author{
François Constantin
}

1 Les processus de construction, consolidation, décomposition, recomposition des espaces sociaux organisés, sont tributaires des formes les plus diverses de solidarité. Les interrogations sur le sens de la quotidienneté qui marquent la fin de ce XXe siècle confirment cette relation, qu'il s'agisse de la construction diffuse de communautés d'usagers d'Internet ou de fondamentalistes religieux (islamistes, évangélistes...), de la remontée de tentations ethno-nationalistes (Caucase, ex-Yougoslavie, Afrique des Grands-Lacs...), de la pérennisation de diasporas discrètement efficaces (Chinois, Indiens...), du renouvellement de circuits parallèles de circulation d'objets de toute nature (devises, armes, drogue ou savonnettes...) aussi bien que de personnes (réfugiés, main-d'oeuvre, touristes...). La vie internationale est ainsi porteuse de messages à lisibilité incertaine dont l'analyse nécessite sans doute un élargissement de la boîte à outils conceptuels. Les messages subliminaux de la vie internationale L'inventaire est inépuisable de ces manifestations, non pas tant de la «crise de l'État » que des limites de l'entreprise associée à sa construction, à savoir l'assignation à résidence territoriale de l'individu dans le but de permettre un meilleur contrôle de ses faits, ses gestes, ses pensées, son identité par le pouvoir politique ${ }^{1}$. Mais la construction normative associée à l'État pouvait poser le principe de la primauté de l'identité national-étatique sur toute autre référence identitaire et établir des frontières ; elle ne pouvait à elle seule abroger les pratiques et représentations propres aux groupes particuliers et aux individus confrontés à cette construction ; l'emploi de la violence (fut-elle légitime) a peut être parfois permis l'anéantissement de certaines d'entre elles mais l'évolution de l'Europe orientale communiste montre la difficulté d'une éradication totale, confirmant qu'il n'a jamais pu empêcher le renouvellement de formes d'escapisme territorial et/ou mental, c'est-à-dire la possibilité de se positionner ailleurs. Bien plus, si l'on a pu considérer que l'entreprise de construction étatique et de centralisation du pouvoir s'est appuyée sur les ressources offertes par le progrès technologique (encadrement, propagande), les derniers développements de celui-ci semblent conduire à une inversion des tendances, la banalisation des moyens de circulation rapide et de la communication électronique 
altérant la position privilégiée de l'État aussi bien dans ses capacités d'initiative que de contrôle des flux de biens, d'idées et de personnes. Ce n'est donc pas une révélation étonnante que de constater que l'information quotidienne nous apporte des illustrations d'un renforcement quantitatif et qualitatif des flux traversant les frontières juridiques des États en marge des pratiques et prescriptions de l'ordre intergouvernemental ${ }^{2}$. Faute de pouvoir énumérer ici, l'accumulation des données accréditant cette dynamique, il convient de recommander vivement la lecture attentive de grands quotidiens (pas seulement "nationaux", pas seulement "du soir») et de tirer parti de l'ouverture remarquable offerte par Courrier international, qui évoquent le foisonnement des échanges transgressant les frontières étatiques en marge des procédures officielles et/ou légales. Ces apports factuels confirment les résultats des observations de terrain qui peuvent être faites par l'universitaire ayant choisi comme champ de recherche privilégié l'Afrique sud-saharienne, où l'entêtement des réalités a imposé aux chercheurs l'investigation d'objets sortant des approches scientifiques classiquement légitimes ${ }^{3}$. Mais le thème n'a rien d'exotique, car les pratiques locales auxquelles l'universitaire doit se consacrer l'amènent à connaître des réseaux d'échange inter-universitaire diversifiés tandis que, ouvert à son environnement régional, il peut constater la vitalité de pratiques transfrontalières cultivées par toutes les composantes des populations avoisinantes, les autorités locales n'étant pas les dernières à prendre des initiatives transnationales mettant l'instance étatique devant le fait accompli. Cet ensemble d'observations allant des micro-décisions renouvelées (le passage de la frontière par le travailleur migrant) à des tendances lourdes perceptibles sur la longue durée (la diffusion de maladies) suggère que malgré ses performances, l'institution étatique et les attributs qui lui sont associés ne parviennent pas répondre à eux seuls à l'ensemble des demandes de la société qu'elle a essayé d'enfermer et à lui "apporter les biens publics» (deliver the goods) qu'elle attend, qu'il s'agisse de sécurité physique, de survie, de bien-être, ou de sens, puisqu'il y a démultiplication de dynamiques privées s'inscrivant dans le champ de ce que les économistes ont les premiers appelé «l'informel ». La spécificité de l'informel qui nous intéresse ici réside dans son caractère "transnational ", s'appuyant sur des formes d'identité héritées, inventées, ou réinventées et structurées de manière plus ou moins diffuse en réseaux. Ceux-ci constituent de véritables lieux de pouvoir et, de ce fait, valorisent l'existence d'espaces de référence parallèles, opposés ou complémentaires à l'espace territorial étatique, ce qui conduit à s'interroger sur la pertinence effective de cette composante fondamentale de la construction étatique en cette fin du millénaire qui avait vu son invention. La question est d'importance dans la mesure où ces espaces de repérage sinon nouveaux, du moins nouvellement reconnus, affectent directement la quotidienneté de quelques centaines de millions de personnes à travers le monde, couvrant aussi bien les zones les plus défavorisées (pauvreté, guerre civile...) que les paradis fiscaux (escapisme fiscal, blanchiment de narco-dollars), impliquant toutes les catégories sociales (du manoeuvre au chef d'industrie, du paysan à l'intellectuel, du pèlerin au guide religieux, du touriste aux gouvernants eux-mêmes) en quête de rémunérations vitales ou superfétatoires pouvant être d'ordre alimentaire, physique, matériel, affectif aussi bien que spirituel. En outre, les données disponibles quant aux chiffres d'affaires induits par ces activités confirment l'importance qu'il convient à accorder à ces dynamiques, en particulier lorsque l'analyse de la vie internationale valorise l'approche économico-financière ${ }^{4}$. Il convient donc de réintégrer dans cette analyse des pratiques sociales parfois peu visibles parce que modestes, cachées ou 
parce que l'on se refuse à les voir, et donc rarement prises en considération par les experts des relations internationales malgré l'ancienneté de ces pratiques constitutives de la vie sociale (besoins d'échanges) bien antérieures à l'État. Certes, la tendance à la subliminalité de l'objet pose le problème des outils permettant d'y accéder.

2 Les outils de l'analyse politique La compréhension plus complète de la vie internationale impose que l'on explore les modalités de ces transactions produites par le télescopage de dynamiques dont chacune, dans son domaine, apparaît encore comme novatrice (ce qui laisse posé le problème de leur reconnaissance comme problématique légitime), à savoir l'informel des économistes, la transnationalité des internationalistes, et les réseaux que revendiquent différentes disciplines des sciences sociales, mais aussi de la communication. L'idée d'analyser des phénomènes politiques subliminaux ne devrait pas apparaître comme une originalité. Elle a été expérimentée, à partir du contexte africain notamment, au travers de l'étude des modes populaires d'action politique animée par Jean-François Bayart ${ }^{5}$. Ceux-ci montraient et la nécessité et la possibilité d'utiliser différents outils conceptuels empruntés à diverses disciplines afin d'arriver à une compréhension plus fine du phénomène crucial qu'est le pouvoir politique et cela où qu'il se manifeste, au Sud certes, point de départ de notre démarche, mais aussi à l'Ouest, et certainement à l'Est, où l'effondrement d'un système réputé totalitaire doit quelque chose à ces dynamiques diffuses. L'extension de la démarche au-delà de l'espace politique interne est pourtant restée limitée ${ }^{6}$. L'une des raisons de cette prudence est sans doute qu'étendre au champ de l'international la réflexion sur les modes populaires d'action politique (devenu alors MPAI) suppose que l'on admette d'abord que ce champ est un espace d'enjeux de pouvoir politique (ce qui s'oppose à une vision du politique liée à l'existence d'un ordre centralisé) et que d'autre part, il ne se réduit pas à des confrontations entre volontés gouvernementales (entre États), et donc qu'il ressort du champ de l'analyse politique, ce qui n'est pas évident pour tout le monde. Mais alors, l'observation de ces pratiques (que les tentatives contemporaines de revalorisation de l'institution étatique sinon dans la pratique, du moins parmi les intellectuels, n'abolissent évidemment pas) conduit à la rencontre de situations dont le traitement procède d'outillages encore peu expérimentés ou appartenant à d'autres formes d'expertise, mais dont surtout la configuration est encore si incertaine que les puristes pourront y voir des concepts (?) à définitions molles ou, à tout le moins, controversées. Pour l'internationaliste, le doute ne devait plus exister sur la signification de la transnationalité. Vingt ans d'investigations directes ou indirectes, avouées ou latentes, pratiquées de R. Keohane à B. Badie et M.C Smouts, permettent d'en cerner l'objet. Il s'agit de : «toute relation qui, par volonté délibérée ou par destination, se construit dans l'espace mondial au-delà du cadre étatique national et qui se réalise en échappant au moins partiellement au contrôle ou à l'action médiatrice des États " ${ }^{7}$. Hormis cette ultime référence traditionnaliste à l'abstraction anthropomorphisée qu'est l'État alors qu'il eut été préférable de viser les "gouvernements", cette définition met bien en évidence l'extraordinaire richesse de l'objet sans pour autant aboutir à sa dilution totale, puisqu'un clivage radical est établi d'une part avec ce qui est de l'ordre de l'intergouvernementalité, d'autre part avec ce qui est de l'ordre de la territorialité étatique; l'une et l'autre se trouvent transcendées sinon subverties, par la transnationalité. La même décennie 70 qui vit émerger la transnationalité avait vu apparaître dans le champ de l'analyse économique la référence à l'informel. Le problème est que le succès du qualificatif depuis qu'il fut utilisé par le Bureau International du Travail (qui l'avait peut être emprunté à K. Hart) 
pour parler du Kenya n'a pas contribué à clarifier sa définition. Toutefois, suivant la présentation de B. Lautier, deux traits paraissent largement partagés, à savoir la petite taille de l'unité de base de l'action, et un fonctionnement en marge de la loi ${ }^{8}$. Suivant cette piste, notre effort d'analyse de l'informel internationalisé laisse de côté les manifestations les plus structurées de la transnationalité, telles que les grandes firmes multinationales ou les églises institutionnalisées, sans pour autant éviter les problèmes de confins auxquels se heurte toute réflexion sur l'informel, confins sur lesquels évoluent habilement nombre d'entreprises familiales transnationales faite de la juxtaposition d'unité aux "petites dimensions » dont la fluidité apparente n'empêche pas une remarquable efficacité productive et capitaliste. La définition de l'informalité est ainsi compliquée par la diversité des manifestations du phénomène, ce qui a conduit à une démultiplication des appellations pas tout à fait synonymes, mais nullement étrangères, au travers des qualificatifs de "clandestin ", "parallèle », "illégal », "secret", "spontané ", ou encore de "seconde économie " ${ }^{9}$; pour ne pas parler des qualificatifs plus spécialisés ressortissant des langages codés propres aux experts d'une forme d'informalité ou d'une zone géographique, comme le magendo est-africain. Les controverses sémantiques ne sont pas négligeables. Elles illustrent au moins pour nous la richesse de l'imagination sociale lorsqu'il s'agit de contourner, pour des raisons plus ou moins bonnes, les régulations étatiques affectant les activités socio-économiques, c'est à dire tout ce qui ramène, de près ou de loin, à la survie. Le propre de l'informel est que son efficacité est souvent liée à sa structuration, peut-être fort éloignée de l'idéal bureaucratique weberien ou de l'organisation scientifique du travail d'H. Simon, mais néanmoins parfaitement rationnelle au regard des objectifs recherchés. Ce n'est sans doute pas un hasard si, de même que l'on a vu apparaître à peu près à la même époque la prise en considération de l'informel et de la transnationalité, celle-ci a eu pour conséquence de faire sortir le concept de réseau hors du champ de la seule communication, qu'il s'agisse des chemins de fer, de la distribution électrique ou de l'électronique, et de permettre son entrée (amorcée à la fin des années 50) dans celui des sciences sociales, de la gestion à l'anthropologie, pour devenir l'épicentre de controverses conceptuelles susceptibles de faire oublier la pertinence de l'outil. Sa nouveauté toute relative et son utilisation par des experts de disciplines fort différenciées des sciences sociales et humaines entretiennent certes un flou que l'esprit scientifique traditionnaliste condamne, mais qui n'altère pas la valeur heuristique du concept, ainsi que le révèle le panorama général présenté par $\mathrm{A}$. Colonomos sous le prétexte du commentaire de l'ouvrage de référence de A. Degenne et $\mathrm{M}$. Forsé ${ }^{10}$. Il est vrai que pour le spécialiste de la vie politique locale, il n'était pas difficile de replacer son analyse ponctuelle dans la catégorie plus large des réseaux politiques, que J. Lagroye définit comme " un système de relations traversant (...) divers secteurs de la vie sociale (politique, économique, culturel, administratif, etc...) et liant durablement ses membres par l'intérêt commun qu'ils trouvent à s'épauler mutuellement dans l'action politique». Et, citant M. Abelès, il précise qu'il s'agit d'un «faisceau d'obligations réciproques et qui fonctionnent sur la longue durée " ${ }^{11}$. On pense irrésistiblement au clientélisme qui nous était apparu transposable de l'espace local à l'espace international. ${ }^{12}$, anticipant ainsi sur une approche élargie en termes de réseaux qu'accréditent la démultiplication et l'influence croissante sur la scène internationale de ces systèmes relationnels multifonctionnels, durables, et dont l'organisation est pourtant caractérisée par un faible degré d'institutionnalisation. Ce dernier critère évite de diluer à l'excès le sens du terme, tout en sachant bien qu'une 
structure institutionnalisée peut être doublée par un système de relations informelles. En d'autres termes, il nous paraît inévitable d'associer informalité et réseau, les réseaux informels pouvant revêtir une ampleur les conduisant à développer leur action au-delà des cadres politiques formels, et en particulier au-delà des limites du territoire national-étatique. Le risque est alors de schématiser à l'excès et d'aboutir à de stériles manichéismes qui opposeraient des réseaux populaires et des relations intergouvernementales (les réseaux peuvent impliquer des élites et être causes d'exploitation sordide, et les rapports intergouvernementaux peuvent intervenir pour lutter contre cette exploitation, quitte à recourir à la voie de l'informel), la logique des réseaux et la logique étatique (chacune peut être amenée à s'appuyer sur l'autre), l'espace des réseaux et le territoire étatique (les membres des réseaux peuvent se situer «ailleurs », ce qui n'est réalisable que parce qu'il y a un «ici », délimité par des frontières). Il est évident que les dynamiques des réseaux informels, produits de certains «choix d'identité", signifient l'existence d'espaces vécus, "perçus à la fois comme lieu qui fournit les conditions matérielles de la vie et la reproduction de la communauté, (...) comme un lieu marqué par des formes de sociabilité particulières (...) et comme un lieu de pouvoir ${ }^{13}$. Ces espaces sont dès lors dotés de plus de sens, d'affectivité, de fonctionnalité et donc d'allégeance que l'espace de l'État qui se trouve ainsi dévalorisé, ridiculisé ou subverti dans son essence et dans son objet, mais jamais totalement ignoré. La logique des réseaux transnationaux consiste à détourner le sens, les fonctions de la composante essentielle du territoire qu'est la frontière pour en faire une simple ressource au service des objectifs poursuivis par les membres des réseaux. L'essentiel n'est donc pas le territoire en tant que tel, mais la présence de frontière, réalité imaginaire, dans le sens où son existence matérielle est un fait actuel, mais où elle peut être ailleurs que là où elle est (voir l'histoire), où elle peut être pensée de différentes façons (ligne, confins, projet...) ou même ne pas être pensée du tout (l'abrogation des frontières est possible, lors de processus d'unification) par l'être pensant qu'est l'homme. L'informel transnational, ou la négation du territoire A un premier degré de l'analyse, l'informel se situe contre l'État en tant qu'espace délimité (territoire) et attributeur d'identité (nationalité), l'un et l'autre considérés comme essentiels puisque la réalité de la prétention des gouvernements à l'exercice de la souveraineté dépend de l'effectivité de leur primauté. Cette prétention, que la théorie juridique et le processus d'institutionnalisation attribuent à l'État, ne suffit pas à entraîner l'éradication des possibilités toujours ouvertes pour l'individu de se situer " ailleurs ", de s'identifier sur d'autres registres, en recourant à la pluralité des cartes d'identité jouables dans la vie quotidienne, et dont la mémoire (car ce sont des cartes à mémoire) s'active particulièrement dans les situations de défaillance de l'appareil étatique. L'informel transnational peut ainsi désigner deux formes d'expression de ces défaillances, formes distinctes, mais complémentaires. Il désigne d'une part un ensemble de structures relationnelles construites par des acteurs sociaux afin d'agir dans des situations vécues de désarroi personnel conjoncturel (répression, pauvreté...) ou d'échec d'une forme particulière d'organisation politico-administrative (échec de la greffe étatique, inefficacité, erratisme de l'appareil gouvernemental). Ces structures aboutissent à des transgressions variées de la frontière à des fins de survie. Les réseaux plus ou moins clandestins de recrutement de main d'oeuvre, d'approvisionnement, de trafic de devises ou de cigarettes, de placement de capitaux ou de mobilisation idéologique ou spirituelle illustrent des formes concrètes de négation de la territorialité étatique. Mais l'informel transnational peut aussi viser des situations de 
fait non encore perçues comme des problèmes, existant donc avant même que des acteurs sociaux ne les perçoivent, les prennent en charge, ne mettent en place des structures d'action qui permettent d'objectiver ce qui n'est jusque là qu'un informel virtuel à un moment donné, dans des espaces donnés, dépassant en tout cas les territoires étatiques (malnutrition, épidémies, dégradation $\mathrm{du}$ milieu naturel, continuités culturelles...). Parce qu'il s'agit de problèmes globaux (régionaux, planétaires...) et diffus (l'effet de serre ?...), les gouvernements hésitent à s'engager dans des programmes d'action, d'autant que l'ampleur des enjeux dépasse le cadre territorial étatique et que d'autre part les résultats éventuels ne sont perceptibles qu'à très long terme, ce qui réduit la rentabilité d'un investissement politique et matériel fort pour qui évolue dans le court terme des échéances électorales et de la conservation du pouvoir. Mais les enjeux sont là, et d'autres que les gouvernements vont s'en saisir ${ }^{14}$. C'est là que les deux modalités de l'informel transnational trouvent leur complémentarité. L'inaction ou la pusillanimité des gouvernants laissent le champ libre à l'initiative des particuliers qui vont pallier ce qu'ils vivent comme des carences en inventant des structures d'action qui, cristallisant ainsi une conscience identitaire, peuvent à terme se rapprocher de l'institutionnalisation au travers d'organisations non gouvernementales comme Amnesty International (Droits de l'Homme), le Fonds mondial pour la nature (W.W.F.) ou le Comité Catholique contre la Faim et pour le Développement (C.C.F.D.), O.N.G. dont l'activité évolue constamment aux confins du formel et de l'informel. Devant insister ici sur les manifestations effectives de l'informel transnational, nous aurons tendance à négliger les problématiques virtuelles au profit de l'analyse des réseaux, dont on peut mettre en évidence trois traits caractéristiques avant d'évoquer d'autres caractères plus controversés.

3 La diversité Il serait bien sûr possible d'envisager un inventaire de réseaux informels transnationaux sans prétendre pour autant à l'exhaustivité. Il représenterait à lui seul tout un volume, et il n'est pas certain que l'on puisse arriver pour autant à une typologie satisfaisante, en raison des autres caractères fondamentaux que sont la plurifonctionnalité et l'adaptabilité; la possibilité pour n'importe quel réseau d'intervenir dans plusieurs domaines différents (aide économique, matrimoniale, spirituelle) et, selon les besoins, de changer de configuration (fonctionner au microlocal ou à l'échelle planétaire) rendent en effet précaire tout effort pour les enfermer dans des catégorisations rigides. Ressemblances et différences se télescopent lorsqu'on observe les constructions et les modes de fonctionnement des diasporas chinoises ou indiennes, des solidarités corporatives des cancérologues ou des politologues, des cartels colombiens ou des mafias russes, des émigrés basques ou des réfugiés somaliens, des fondamentalismes islamistes ou des professionnels "sans frontière", du trafic clandestin d'armes ou de cigarettes, des flux d'investissement privés ou de trafic de devises, de solidarités inuits ou de la circulation de sportifs de haut niveau, des antennes paraboliques ou du cyberespace... Ces quelques coups d'éclairage embryonnaires sur divers sites d'observation possibles de l'informel transnational suffisent à mettre en évidence une première modalité de la diversité, affectant directement la question du territoire. Dans ses manifestations classiques, il a pour conséquence de privilégier un espace d'identification différent de celui de l'État, qui peut être celui défini par les réseaux matrimoniaux, par la circulation des devises accumulées, par les chemins de Saint Jacques de Compostelle, de la Mecque, ou par la géographie du Club Méditerranée ou de Frères des Hommes. Ici, des contre-espaces peuvent être dessinés, montrant bien la matérialité de la marge de choix et de mobilité 
conservée par les acteurs sociaux. Deux précisions doivent toutefois être apportées. Tout d'abord, la matérialité territoriale peut être accessoire et de ce fait fluide, l'ancrage territorial du réseau évoluant avec les choix stratégiques et tactiques de tout ou partie de ses composantes; il y a certes une territorialité des réseaux de la drogue, mais sauf en ce qui concerne les lieux de production, nécessairement peu mobiles (mais partiellement hors de toute territorialité connue, comme par exemple les laboratoires de transformation du produit brut), leur espace se déforme en fonction des contraintes et des opportunités créées par l'environnement extérieur, comme par exemple actuellement les conséquences des pressions militaro-policières du gouvernement américain sur les narco-trafiquants latino-américains ou les effets de la déstructuration des espaces étatiques et répressifs est-européens ou africains ${ }^{15}$. A la limite, et c'est là la seconde précision, la référence territoriale s'estompe totalement, le réseau fonctionnant en tant que lien dépourvu de toute matérialité physique à l'image de l'ensemble virtuel constitué par les utilisateurs d'antennes paraboliques ou d'Internet : "Les réseaux n'appartiennent à personne... Il existe désormais un système de cryptographie pratiquement inviolable... Il garantit à ses utilisateurs un parfait anonymat : on ne peut connaître leur identité, leur nationalité, ni même savoir de quel continent ils émettent " ${ }^{16}$. L'évacuation à peu près totale du territoire liée à cette forme de communication créatrice d'une identité totalement délocalisée, sinon alocalisée, rejoint certaines observations faites ici en matière financière par W. Andreff. Mais la diversité ne se réduit pas à des différences de degré dans la matérialité de l'informel transnational. Une autre modalité de différenciation peut s'inscrire dans la différence des positionnements des réseaux le long de la ligne qui joint traditionnalisme et innovation. L'exercice n'est pas sans risque, car la rémanence de réseaux anciens ne peut s'expliquer que parce qu'ils se sont adaptés aux changements des circonstances et savent répondre à des besoins présents. Ici comme dans le domaine de la culture, la tradition est active dans la mesure où elle reçoit et traite les défis actuels. La vitalité des réseaux de parenté ou claniques indiens, arabes ou chinois entretenant des liens entre parents séparés par des milliers de kilomètres et des dizaines d'années d'absence ne s'explique pas par un simple culte de l'habitude, mais par le fait qu'au travers de ce culte de la famille, du clan ou de la caste, des besoins actuels trouvent leur satisfaction ${ }^{17}$. Il importe donc de s'écarter des représentations qui tendent à considérer les réseaux informels (transnationaux ou non) comme de simples survivances, des archaïsmes hérités d'un monde pré-moderne, illustrant le caractère inachevé du procès d'institutionnalisation des relations sociales. Il s'agit au contraire de mécanismes de réponse aux insuffisances des performances des produits de cette institutionnalisation. La distinction tradition/innovation n'est donc pertinente que dans la seule mesure où certains réseaux semblent dépourvus d'ancrage historique traditionnel, que ce soit sous forme de parenté, d'ethnicité, d'intérêt économique ou matériel, de spiritualité ou même à la limite d'héritage culturel: les réseaux postmodernes liés à la maîtrise de la communication électronique identifient des communautés sans profondeur historique et peut être sans avenir durable (du fait de la banalisation possible des procédures), mais avec un présent certain comme le savent les utilisateurs du courrier électronique : « e-mail ». D'autres réseaux situés en apparence dans le même espace-temps, comme ceux traitant la défense des droits de l'Homme, les causes humanitaires ou la réflexion sur l'environnement naturel se situent cependant davantage dans une perspective historique, en tant que continuateur d'une culture humaniste inquiète devant les limites manifestes des capacités de l'Homme à contrôler 
toutes les conséquences de ses actes. L'efficacité propre de l'informel internationalisé fait qu'en la matière, il n'y a pas place pour une querelle des Anciens et des Modernes. On peut aisément imaginer d'autres critères de distinction comme nous avons pu le faire en identifiant les transnationalités «historiques», qui peuvent être des transnationalités de voisinage (petite contrebande transfrontalière, pastoralisme...), de parenté (Indiens, Hadramis, Omani en Afrique de l'Est...) ou des transnationalités de valeur (idéologie religieuse, défense d'une cause humanitaire, éthique...), et des "transnationalités de crise", d'invention en apparence plus conjoncturelle dans la mesure où elles s'inscrivent dans un contexte de dégradation des conditions d'existence, ce qui conduit à constater l'opposition de deux stratégies, l'une visant à organiser la « survie à la crise » (réfugiés, main d'oeuvre migrante), l'autre recherchant la «survie par la crise» et à la limite par l'exploitation des victimes de la crise (contrebande d'armes, drogue, mafias). Mais là encore, les limites entre catégories sont fragiles ${ }^{18}$. La plupart des réseaux identifiés s'inscrivent au moins potentiellement dans un continuum de relations plus ou moins consciemment perçues. Même ceux qui paraissent les plus confinés territorialement (cités-boutiques transfrontalières de par le monde) ne fonctionnent que parce qu'ils sont articulés sur la circulation de produits, de devises et de personnes dépassant largement le micro-cadre local. En somme, leur territorialité n'est qu'une composante particulière d'une stratégie pensée non pas par référence à des territoires, mais par référence aux impératifs de rentabilisation de la circulation d'objets (matériels ou immatériels) associés au réseau ${ }^{19}$. La primauté accordée à l'échange explique aussi la relativité des distinctions que l'on pourrait rechercher à partir du degré de structuration des réseaux. L'informalité n'exclut pas l'existence de structure puisque tout échange renouvelé impose leur existence, qu'il s'agisse des structures de la parenté, des structures du marché financier, de structures d'approvisionnement et de transport, etc... L'informel peut en outre se développer en parallèle de structures de type bureaucratique afin de surmonter leur blocage ou leur inertie. C'est à ce titre que le mode d'action des organismes humanitaires (O.N.G.) se situe souvent sur le registre de l'informel et du réseau (du fait des rapports parfois difficiles avec les autorités publiques), venant relativiser la portée des différences existant entre Médecins du Monde ou la Cimade d'une part et les réseaux de solidarité et d'affaires libanais ou chinois d'autre part. C'est sur la base des modes d'action effectifs, et non sur les apparences formelles, qu'il convient de positionner organismes et réseaux agissant sur la scène internationale, ce qui amène par exemple à placer dans deux catégories différentes des organismes par ailleurs assez proches tels que la Croix Rouge (qui fonctionne uniquement dans le champ formalisé de l'intergouvernemental) et Amnesty International (qui privilégie le recours à des méthodes qui sont de l'ordre de l'informel). L'empirisme qu'impose l'analyse interne de réseaux informels explique les difficultés pour trouver un critère satisfaisant de classification même en se limitant aux réseaux transnationaux. Le critère instrumental (à quoi sert le réseau ?) n'est pas plus opérationnel en raison d'un autre caractère distinctif : la plurifonctionnalité.

4 La plurifonctionnalité S'appuyant sur une analyse de J.M. Rodrigo portant sur le Pérou, B. Lautier indique que « sans être une alternative économique, l'économie populaire est vue comme une alternative sociale, permettant le renouveau d'une démocratie à la base... ${ }^{20}$, formule qui résume en peu de mots la capacité de l'informel à intervenir à la fois dans l'économique, le social et le politique. Et l'énoncé est loin d'être complet, car dans les réseaux transnationaux informels, on peut lire non seulement la recherche de rétributions matérielles au travers de redistributions en nature ou de participation au 
pouvoir (fictive ou effective, mais perçue comme telle par les demandeurs), mais aussi la recherche de dignité, de sens, de prestige ou d'affection. D'une façon ou d'une autre, la pérennisation de l'informel est conditionnée par sa capacité à produire des repères permettant à l'individu (ou à des micro-sociétés) de savoir où il est et pourquoi, et ceci de manière positive, dans la mesure où l'identification au réseau apporte un plus dans son existence. Il serait aussi réducteur de ne voir dans le réseau des Ismaéliens qu'une secte shiite dont l'Aga Khan est le chef spirituel, que de le considérer comme une simple bande de capitalistes à l'affût des placements les plus intéressants à travers le monde. Leur sens des affaires n'est pas usurpé, montrant fort bien que la fidélité à une éthique musulmane n'est incompatible ni avec l'esprit du capitalisme, ni avec l'assimilation des rituels et des mondanités des aristocraties européennes modernes. Mais pour des dizaines de milliers de fidèles, l'Aga Khan est tout autant un chef religieux dont les capacités accumulatrices assurent la prospérité - fut-elle relative - de fidèles pouvant bénéficier de priorités d'embauche dans les établissements dirigés par des Ismaéliens, de soins de santé efficaces dans les hôpitaux financés par des Ismaéliens, de bourses d'étude dans les meilleures institutions anglo-saxonnes ou helvètes, ou d'un traitement de faveur de la part des gouvernements qui doivent beaucoup au dynamisme et à l'entregent de ces petites minorités fortunées. Certes, tous les fidèles ne bénéficient pas de toutes ces prestations terrestres, mais elles leur sont potentiellement ouvertes, et surtout, s'ajoutent pour chacun les prestations spirituelles dispensées par le chef spirituel des septimaniens nizarites que leur marginalité historique a pu familiariser aux pratiques "cachées" de l'informel ${ }^{21}$. On pourrait développer de la même manière la capacité des diasporas chinoises, les Huaqiao, ou libanaises à mener des politiques efficaces de placement immobilier ou à tisser la trame de trafics d'armes impliquant des acteurs multiples au travers de la planète, à organiser les hautes études des fils les plus doués, à appareiller les couples pour le plus grand bonheur des intéressés et du réseau, à accueillir des frères persécutés, à punir des membres du réseau ou des étrangers ayant failli aux usages qui assurent sa cohérence ou tout simplement à financer le développement de la Chine populaire ou la reconstruction du Liban ${ }^{22}$. Les difficultés rencontrées dans la mise en oeuvre de programmes de lutte contre les cartels de la drogue révèlent de même la complexité des interactions qui interviennent dans l'architecture de ces réseaux dont les fonctions économiques, sociales et symboliques se télescopent d'une manière suffisamment informelle pour surmonter les contradictions et entretenir une synthèse permettant à chacun, petit cultivateur ou méprisable parrain, de trouver son compte dans l'entreprise, et donc de lutter pour la soutenir ${ }^{23}$. Cette aptitude des réseaux informels transnationaux à répondre à de multiples besoins individuels et collectifs acquiert une importance nouvelle dans le contexte traumatisant de la "dérégulation » et de la mondialisation. A travers eux, l'individu conserve ou retrouve les repères permettant d'établir et de gérer le lien entre son positionnement propre (le micro-local) et les dynamiques mondialisées qui remettent en cause les repères formels traditionnels que constituaient les cadres étatiques. Par l'intermédiaire du réseau, qu'il s'agisse d'une secte, d'un système de parenté, des branchés d'Internet, ou d'opérateurs sur les marchés financiers, différents espaces de référence se concrétisent, permettant de surmonter les troubles inhérents aux sentiments d'insécurité et d'incertitude consécutifs au retrait de l'État, ce qui conduit à la survalorisation de ces créations extra-étatiques. Bien plus, cette identification à la confrérie, au cartel ou à l'entreprise, en marge des réglementations gouvernementales, peut être perçue comme s'inscrivant 
dans le sens de l'idéologie contemporaine de la post-modernité, privilégiant les démarches, les initiatives performantes indépendantes des aides publiques. L'informel transnational est donc une contribution à l'effectivité de "grands marchés » ouverts ; le honteux (le commerce clandestin, représentant par exemple largement la moitié du commerce effectif en Afrique sud-saharienne) acquiert une légitimité de pointe, puisqu'étant à l'avant-garde de l'esprit d'initiative, du marché libre, il s'inscrit parfaitement dans l'idéologie correcte du nouvel ordre international. Selon les propos attribués au Japonais R. Takeoka, "l'Asie a déjà son grand marché, mais il est parallèle ${ }^{24}$. On doit enfin préciser que cette plurifonctionnalité peut être aussi bien simultanée que successive. La simultanéité signifie qu'au même instant, au travers d'une même action, le fonctionnement du réseau permet aux différents intervenants de trouver des satisfactions de nature différente (affectives, religieuses, matérielles, physiques) et d'intensité différente (besoin vital pour celui confronté à la famine ou à des persécutions, besoin marginal pour celui qui, accordant un secours, et/ou conforte la fidélité d'un disciple, accroît son prestige...). Mais la plurifonctionnalité peut aussi s'exprimer de manière successive, au travers de l'aptitude du réseau à se reconvertir, ce qui ramène un autre trait essentiel associé à l'informalité, la souplesse.

5 Souplesse et adaptabilité Même si l'informel ne peut être efficace que parce qu'il s'appuie sur de solides structures assurant la cohésion du réseau, les rigidités relatives qu'elles peuvent introduire (par ex. la fidélité clanique, la loi du milieu, l'esprit de caste, les règles de redistribution des profits,...) laissent ouverte la possibilité de réagir rapidement en fonction des changements de la conjoncture dans tous ses aspects, c'est à dire aussi bien politique (coups d'État, renforcement des contrôles), économique (variation des taux d'intérêt, famine), sociale (crise de l'emploi, fermeture d'universités), technologique (inventions de nouveaux procédés) ou culturelle (persécutions religieuses), que locales (catastrophe naturelle), nationales (changement d'orientation politique) ou internationales (effondrement du bloc communiste). Les composantes du réseau peuvent ainsi mettre l'accent sur l'action d'entraide sociale en période de troubles menaçant des membres du réseau (décision d'Idi Amin d'expulser les Indiens d'Ouganda), puis sur les capacités d'investissement lorsqu'une demande forte laisse augurer de bons placements (négociation des successeurs d'Idi Amin Dada avec quelques familles indiennes pour qu'elles réinvestissent en Ouganda), ou en fonction des variations de la demande (forte) de produits dont l'offre est rare sur le marché officiel (électronique, armes, sécurité, whisky, salut de l'âme...). L'efficacité des systèmes informels peut dépendre aussi de leur aptitude à être présent aux lieux stratégiques et mieux encore, de les contrôler et d'agir ainsi aussi bien à la base, sur le terrain, auprès des masses (le boutiquier indien ou chinois disponible à toute heure et pour tout service, le mouride sur les marchés forains, le changeur de rue...) qu'au sommet, dans les salons des palais présidentiels (le grand banquier, qui peut être chinois, indien, libanais, arabe... ou luxembourgeois, le patron de la multinationale familiale, le calife ou le cardinal, le notable catalan ou arabo-est africain...). Autrement dit, l'efficacité du réseau repose sur la diversité de ses membres, notamment du point de vue de leurs aptitudes à maîtriser les technologies les plus diverses de l'échange, de la communication, de la prise de décision et de l'action, soit en bricolant des microsolutions locales (organiser le départ de main d'oeuvre, prêter de l'argent), soit en conduisant des actions de lobbying (auprès du gouverneur de province, du ministre, du chef d'état major, de la Banque mondiale), soit en assimilant les formes les plus élaborées de la micro-informatique et des circuits électroniques. Chacun à son niveau 
joue un rôle crucial dans le système, car plus le réseau est capable d'évoluer sur différents registres, sur différents espaces (y compris si nécessaire sur le registre institutionnel), meilleures sont ses chances d'exploiter à son profit les ressources de son environnement. Cette fluidité renforce aussi ses chances d'échapper aux tentatives de prise de contrôle par l'appareil politico-administratif d'État. L'informel transnational est, par ce caractère, plus qu'un système de défense efficace pour les groupes sociaux en situation de précarité parce qu'ils sont minoritaires ou marginaux au sein des systèmes étatiques. C'est la raison pour laquelle la stratégie cultivée par les diasporas, des Juifs aux Arméniens, est aujourd'hui reproduite par d'autres communautés qui se considèrent, à tort ou à raison, comme oubliées, brimées ou opprimées par les institutions étatiques. Ceci explique la constitution d'un réseau circumpolaire des Inuits de l'Alaska à la Russie en passant par le Canada et le Groenland ${ }^{25} \mathrm{ou}$, dans un contexte où l'emprise des structures bureaucratiques est pourtant autrement plus intégrée techniquement et culturellement, la constitution d'« euro-régions » visant à faire pression sur les politiques intergouvernementales, à l'image de « l'arc Sud-Europe Atlantique » imaginé pour lutter contre l'hégémonie de la «banane bleue» (Londres, Rhin, Milan) au sein de l'Union européenne ou de l'«Internazionale Bodenseekonferenz " liant des cantons suisses et des Länder allemands et autrichiens riverains du lac de Constance, mais négligés par les grandes métropoles environnantes ${ }^{26}$. L'adaptabilité apparaît ainsi comme le produit de l'adversité qui a imposé la nécessité, pour un groupe opprimé, de faire preuve d'imagination, d'initiative, de prise de risque pour au minimum, survivre, retrouver une dignité, et mieux encore, se repositionner en position de force sur la scène internationale, contournant ainsi l'ingratitude et/ou les menaces supposées ou réelles des gouvernements étatiques et ouvrant des possibilités d'action et de profit élargies. La survie et la revanche éventuelle de groupes persécutés passe par cette démarche, discrète dans ses manifestations extérieures, mais qui a fait la réputation légendaire, mais nullement mythique, des entrepreneurs exilés chinois, libanais ou indiens, et que renouvellent à une échelle plus modeste courtiers yoruba et haoussa en Afrique de l'Ouest, « Nana-Benz » togolaises ou Vietkieu d'origine vietnamienne en Asie du Sud$\mathrm{Est}^{27}{ }^{28}$. D'autres caractères sont parfois évoqués, comme les tendances endogamiques des réseaux, ou l'image populaire, anti-élitaire souvent associée à l'informalité. Leur pertinence peut être toutefois discutée. Dans la mesure où les systèmes informels peuvent être porteurs d'un sentiment identitaire fort, balisé par un ensemble de valeurs éthiques ou religieuses nettement circonscrites, leur pénétration suppose effectivement l'accomplissement d'un parcours initiatique permettant de tester la fiabilité du néophyte. Il est vrai que dans certains cas, le recrutement est strictement endogamique, en raison notamment de la rigidité des structures de caste, de clan ou de manière déjà plus floue, en raison de la sublimation d'un référentiel comme l'origine régionale. La soumission aux règles d'un dogme intransigeant (interprétation de la Bible ou du Coran) est une autre modalité de filtrage qui, pour être stricte, permet néanmoins d'ouvrir le réseau à des apports extérieurs (extension du wahhabisme). Cette prudence s'explique encore par le fait que la réussite d'un système informel passe non seulement par la discrétion de ses manoeuvres, mais aussi par l'établissement de liens de confiance absolue sans laquelle la cohésion de toute structure fluide serait d'autant plus précaire qu'elle se trouve spatialement dispersée, ce qui est en général le propre des réseaux transnationaux. On ne saurait enfin oublier l'attitude des sociétés d'accueil ou de leur gouvernement qui, par des pratiques discriminatoires ou des 
agressions verbales ou physiques, entretiennent la méfiance des victimes de cet ostracisme à l'égard de leurs voisins et le choix obligé de rester entre soi. Indiens du Kenya, d'Ouganda ou de Tanzanie connaissent le problème, tout comme les chinois expatriés ou les maghrébins en Europe. L'efficacité de l'informel transnational placé dans un environnement lui-même peu ouvert impose généralement l'extrême prudence dans le recrutement des partenaires, ce qui explique l'importance conservée par les relations de parenté (fut-elle élargie ou artificielle) dans l'architecture de bon nombre de réseaux. L'adhésion au code commun de valeurs est d'autant plus nécessaire que non seulement le système est dispersé mais qu'en outre, il est généralement solidement hiérarchisé, donc inégalitaire. La tendance générale qui veut que l'informel s'oppose au gouvernemental a pu conduire à y voir une forme de mode populaire d'action pas seulement politique. Il est parfois effectivement le fait d'individus modestes, ou le produit d'initiatives de leaders de conscience ou d'opinion cherchant par là à aider des groupes fragilisés, comme les réfugiés, les minorités en exil, les victimes de fléaux naturels, politiques ou économiques. $\mathrm{Si}$, comme nous le pensons, l'action transnationale des O.N.G. passe pour partie par la voie de l'informel, elle accrédite la dimension populaire de celui-ci. De même, lorsque l'on évoque la transnationalité informelle de voisinage ou les bricolages inventés par des particuliers pour assurer leur survie, la main d'oeuvre migrante, elle est alors l'affaire de ceux que P. Sansot appelle, dans un autre contexte, les gens de peu ${ }^{29}$. Mais lorsqu'on évoque les cartels de la drogue, les mafias, ou plus légalement les milliards de dollars que font circuler à travers l'Asie du Sud-Est les hommes d'affaires huaqiao, il s'agit de toute autre chose. La structure de ces réseaux, aussi informels soient-ils, est fortement hiérarchisée, que l'autorité suprême soit celle d'un «caïd», d'un gourou, d'un patriarche, ou d'un faux vrai-pdg. En tant que système d'action concertée, un réseau informel est nécessairement un système d'autorité, ce qui, dès lors que l'on dépasse la micro-décision individuelle, induit l'inégalité (clivage décideur-exécutant), inégalité que le code de valeur propre au réseau s'applique à légitimer, et donc à relativiser. Son intensité varie par conséquent avec l'ampleur de l'espace d'action du réseau, les réseaux transnationaux étant de ce fait particulièrement concernés. Cependant, la souplesse du réseau peut atténuer la perception de la domination dans la mesure où elle signifie la possibilité de fortes décentralisations des initiatives, sinon un véritable polycentrisme. Tout dépend en particulier de l'amplitude des actions entreprises (voisinage, immédiateté/longue distance, longue durée), des moyens à mobiliser (petits marchés, transports usuels/haute finance, hautes technologies) et de l'importance des dividendes attendus (humanitaire/trafic d'armes ou d'ivoire). L'efficacité de réseaux lourds, théâtres d'enjeux de pouvoir (celui de l'argent) importants, impose une solide structuration des relations de pouvoir entre les différentes composantes du réseau, un accès à des centres stratégiques de décision nationaux ou internationaux, et entraîne une très inégalitaire distribution de l'information et des revenus retirés de la transgression informelle de la frontière. La démocratisation apparente de vecteurs de transnationalité informelle, comme le réseau Internet, échappe d'autant moins à ce type d'interrogation que la distribution du pouvoir y est diffuse, (production de serveurs, financements), posant notamment le problème de la liberté d'expression ${ }^{30}$. L'informel, par sa nature même, favorise les représentations imaginaires les plus extrêmes, des plus généreuses aux plus machiavéliques débordant sans doute sa réalité qui est plurielle. Les images contrastées de stimulant provocateur ou de méprisable exploiteur trouvent notamment leur origine dans la possibilité qu'il a de pratiquer une 
véritable déterritorialisation, c'est-à-dire d'échapper à l'enfermement territorial, parce que leur champ d'activité est indifférencié et se définit par l'implantation des membres du réseau (et non l'inverse, comme dans la logique étatique) qui peuvent se déplacer au gré des circonstances des Caraïbes à la Scandinavie ou à l'Australie, et parce que ce champ peut être modulé en fonction de la demande interne au réseau ou de contraintes environnementales (intervenir au niveau micro, agir à l'échelle macro). La prospérité de l'informel transnational dépend de la présence non pas de territoires étatiques en tant que tels, mais de différentiels de situations (politique, économique, sociale, culturelle, etc...) provoqués en particulier par l'existence de frontières et qui peuvent être exploitées avec, hors ou contre les régulations gouvernementales. Ce qui n'exclut nullement les rétroactions. De la subversion du territoire à la récupération de l'informel La transformation de la frontière en ressource (politique, sociale, commerciale...) selon des modalités différentes (contournement, évitement, corruption, sinon même réappropriation) implique que l'on reconnaisse son existence; dire que l'informel «nie » la frontière n'est qu'une image. Bien plus, dès lors que la frontière est traitée comme une ressource, elle devient utile, sinon nécessaire. Sans elle, en effet, le réseau peut se trouver menacé dans ses performances (que ce soit pour la sécurité de ses membres ou pour les bénéfices tirés de la contrebande), et donc dans son existence même. Ainsi, les recompositions auxquelles donnent lieu des entreprises comme la construction européenne ou d'autres formes d'intégration régionale (l'Amérique de nord avec l'A.L.E.N.A., l'Asie du Sud-Est avec l'A.S.E.A.N., l'Afrique de l'Ouest ou l'Afrique australe) constituent des défis pour les activités informelles transnationales qui peuvent être obligés de se redéployer, sinon de se reconvertir. Mais dans ce type de situation, les initiatives gouvernementales peuvent apparaître comme une reconnaissance des limites de leurs capacités à contrôler leur espace national-étatique, les dynamiques transnationales leur ayant imposé le développement de la concertation intergouvernementale. A l'inverse, la déconstruction d'espaces comme l'ancienne U.R.S.S. et l'ensemble du bloc de l'Est (en tant que «bloc») en démultipliant les frontières, a créé de nouvelles opportunités sur le terrain de l'informel ${ }^{31}$. L'informel transnational se situe moins contre le territoire étatique que contre l'institution étatique à laquelle il essaie d'échapper (ou qu'il essaie même de subjuguer) parce qu'il apparaît comme un appareil de pouvoir illégitime, soit parce qu'il est trop efficace (contrôle policier, contrôles bureaucratiques, répression...) soit parce qu'il ne l'est pas assez (insécurité, pénuries...). C'est ce contexte qui fait de la frontière une ressource majeure, et de ce fait un enjeu susceptible d'effets pervers en cascade. Les agents de l'État dont la fonction est dans l'existence et la non-subversion de la frontière, découvrant et le besoin de frontières subversibles, et l'impossibilité de la contrôler parfaitement, pourront monnayer sa transgression. S'ils piègent ainsi le réseau, ils se trouvent eux mêmes piégés puisqu'ils entrent dans un système informel de corruption susceptible de déstabiliser l'ensemble de l'appareil politico-administratif.

6 La frontière comme ressource de l'informel transnational L'informel transnational fonctionne non par sur la base de territoires, mais sur la base de situations que la présence de frontières influence positivement ou négativement. Les incidences de la frontière sont positives pour les acteurs transnationaux dans la mesure où elle crée des différences de contexte qui habilement gérées, peuvent être sources d'avantages qui activent et justifient la dynamique du réseau. $C^{\prime}$ est en ce sens que la frontière est une ressource car elle s'inscrit dans une relation d'échange. De ce fait, la distinction que l'on pourrait envisager entre États forts, bureaucratisés, disposant effectivement du 
monopole de la contrainte légitime, et États " mous ", sous développés ou en situation de guerre civile ouverte ou de déstructuration profonde, s'avère peu pertinente. Elle introduit certes des différences dans l'intensité et les modalités de la subversion de la frontière, parce que les opportunités créées ne sont pas les mêmes. Mais alors, ce ne sont pas les performances institutionnelles de la structure étatique qui sont en cause, mais plus fondamentalement la nature des activités qui se développent à l'intérieur des frontières. Le clivage théorique de référence est alors celui distinguant développement et sous-développement, avec toutes les ambiguïtés accompagnant ces termes qui ne changent rien au fait que pour tous, la frontière peut être une ressource. Ainsi les pays du Nord développés sont-ils particulièrement exposés aux pressions des réseaux de migration de main d'oeuvre pour qui le franchissement de la frontière signifie l'accès à des opportunités qui doivent rendre les conditions d'existence moins précaires. L'actualité est ainsi riche des péripéties et des incidences de flux qui ont été longtemps orientés essentiellement Sud-Nord (Afrique-Europe occidentale, Turquie-Europe occidentale, Viet-Nam-Europe de l'Est, Amérique latine-Amérique du nord) et qui sont aujourd'hui concurrencés par une circulation Est-Ouest (Europe de l'Est-Europe de l'Ouest). Ils attirent l'attention sur la permanence de circuits régionalisés de migration des travailleurs aussi bien dans des situations de voisinage (des États enclavés vers les États côtiers en Afrique de l'Ouest, de la côte orientale d'Afrique vers la péninsule arabe, des États d'Afrique australe vers l'Afrique du sud) qu'à longue distance (Indiens, Pakistanais, Philippins vers les Émirats du Golfe). Cette circulation de personnes vers des régions réputées plus prospères est un objet d'études classiques ${ }^{32}$. De même, la richesse relative de l'Europe occidentale, de l'Amérique du Nord, de l'Australie ou du Japon en fait des lieux privilégiés de destination et de circulation de profits accumulés soit à partir d'activités à caractère rentier (placements de quelque 2000 milliards $\$$ de fonds de pension sur les marchés financiers mondiaux, ou encore des pétrodollars dans l'immobilier européen ou nord-américain), soit à partir de l'exploitation de populations dépendantes (faibles salaires, absence de protection sociale), soit d'activités à caractère criminel. Si l'institutionnalisation signifie l'abstraction, l'institutionnalisation parfaite $\mathrm{du}$ système bancaire suisse, en fait une destination privilégiée pour des placements en quête d'anonymat, confirmant ainsi notre hypothèse qu'accréditait le constat de la prolifération de petits trafics prospères (de «jeans» ou de dollars) dans des États réputés très bureaucratisés et totalitaires (U.R.S.S., R.D.A....). Un degré élevé d'institutionnalisation, d'autoritarisme, de développement économique et social n'est pas un facteur de blocage des tentations à l'informalité transnationale. Bien au contraire, ce sont les différenciations nées de la simultanéité de différences de degrés dans ce qui n'est jamais que relatif (institutionnalisation, développement, libéralisation, croissance...) qui activent une dialectique demande-offre qui affecte toutes les composantes du système international. Les trafics de narcotiques constituent l'illustration extrême de cette vulnérabilité généralisée des entités étatiques puisqu'ils impliquent en même temps la pauvreté des vallées andines ou indiennes, la fragilité des contrôles douaniers colombiens, nigérians ou kenyans, la prospérité de familles ou de banques nord-américaines ou basés dans des micro États caraïbes, et la rigueur formelle des règles édictées par de petits Grands duchés ou quelque confédération montagnarde. La frontière n'est pas seulement une ressource parce qu'elle permet d'échapper à des poursuites, des contrôles, des privations ou des sanctions. En général, parce qu'elle est effectivement un moyen de trouver protection, elle est directement génératrice de plus-values. Son passage en marge de la légalité implique une prise de 
risque de la part de l'opérateur (le passeur, l'organisateur du système) qui peut être rémunérée à la mesure des dangers encourus (évasion d'opposants politiques), mais aussi à la mesure des profits attendus, compte tenu de l'état du marché ${ }^{33}$; dans un contexte de pénurie avancée, l'intégration dans des réseaux de trafics de stupéfiants est plus rénumératrice (malgré et à cause des risques) que la participation à la contrebande de sacs de maïs, qui peut être certes source de bénéfices non-imposables importants, mais à condition de convoyer des quantités encombrantes et moins discrètes que la poudre blanche ${ }^{34}$. Le caractère de ressource associé à la frontière suppose que sa subversion soit rentable. L'ampleur des plus-values qu'elle peut générer est donc fonction de l'ampleur de la demande sur le marché, demande éminemment fluctuante et diversifiée. Elle sera d'autant plus forte que le réseau peut être capable d'atteindre un marché élargi, éventuellement planétaire et d'entretenir la demande. Trafic d'armes comme trafic de drogue ont ceci de commun qu'ils s'appliquent à créer et à entretenir une dépendance du client qui permet des bénéfices durables. Mais là encore, le concept de rémunération ne doit pas être entendu en termes strictement matériels. Il peut viser aussi des rémunérations symboliques ou spirituelles (prestige social, salut de l'âme) dont la valeur marchande aléatoire n'affecte nullement leur importance dans une relation de pouvoir. Faire la preuve de sa capacité à jouer avec les frontières est un facteur de distinction sociale dont peuvent jouer l'homme d'affaire, l'entrepreneur, l'universitaire ou le prophète qui, évoluant dans des espaces transnationaux, montrent qu'ils sont chez eux partout et qu'ils sont donc importants. Quelles que soient la nature et l'ampleur des rétributions attendues, la frontière se trouve donc réduite à l'état abstrait de valeur marchande, composante indispensable à la dynamique de réseaux informels transnationaux. Dans certaines situations cependant, elle constitue plutôt une entrave à la constitution ou à la reconstruction de solidarités identitaires perdues ou menacées. C'est dans ce cas seulement que l'informel transnational peut avoir pour perspective la négation totale, l'élimination de la frontière. Si elle est ressource, elle est perçue comme étant au service d'un pouvoir étatique illégitime, parce qu'il empêche la réunion, sinon la fusion de populations partageant une unité considérée comme fondamentale, qui peut être d'ordre ethnique (les Kurdes, les Irlandais), culturel (les Basques) ou religieux (confréries soufi ou églises chrétiennes). En tant que ressource du pouvoir central, la frontière peut se trouver contestée non seulement par des groupes privés, mais aussi par des autorités locales qui militent sinon pour leur abolition, du moins pour l'atténuation de leur importance, leur déplacement ou leur changement de signification, ce qui revient toujours à remettre en cause l'objet du territoire et donc les fonctions de l'institution étatique ${ }^{35}$. Toutefois, dès lors que la frontière est contestée, ou qu'elle est détournée de ses fins, l'autorité qui en est gardienne, à savoir le gouvernement au travers de ses agents publics, peut retourner la situation en entrant dans le jeu même de l'informel transnational. L'informel transnational comme ressource diplomatique Le jeu de l'informel transnational laisse rarement indifférents les gouvernements, ou s'ils feignent de l'être, c'est en toute hypothèse un choix politique délibéré signifiant soit la résignation devant une sorte d'inévitable répartition des tâches entre un secteur officiel qui ne peut pas répondre à toutes les demandes et qui ne peut pas tout contrôler et un secteur non-officiel exploitant et palliant ces défaillances, soit l'infiltration et la récupération du réseau pour le compte de la politique gouvernementale, soit enfin la compromission à des fins personnelles conformément à une stratégie d'ordre néo patrimonial ${ }^{36}$. Mais quelle que soit l'hypothèse retenue, la territorialité officielle se trouve perturbée, démentie, repensée. 
Sur la scène publique, l'attitude affichée des gouvernants vise d'abord à réagir afin de faire respecter leurs compétences territoriales. Ils peuvent aussi songer à retourner l'activité des réseaux informels transnationaux à leur profit. Mais dans un cas comme dans l'autre, celà revient de leur part à reconnaitre l'importance des initiatives prises en violation de la souveraineté territoriale. La réaction défensive consiste donc à essayer d'imposer un recadrage des activités transnationales pour les mettre en conformité avec la réglementation en vigueur sur le territoire où se développent ces activités, ceci par un renforcement des contrôles aux frontières et dans tous les lieux de transit de l'informel transnational, c'est-à-dire les ports, aéroports, établissements commerciaux, bancaires, financiers, mais aussi les trottoirs, coins de rue et arrières boutiques des grandes villes, de Casablanca à Zanzibar, de Medellin à Manille, de Roissy à Saint Petersbourg ${ }^{37}$. L'outillage des mesures répressives de la compétence des gouvernements est mis en oeuvre pour paralyser le fonctionnement de réseaux porteurs de subversion politique (d'où l'expulsion de ressortissants libyens ou iraniens), sociale (d'où la répression des ateliers clandestins dans le XIIIe arrondissement parisien), morale ( $\mathrm{d}$ 'où la condamnation de voyagistes spéciaux spécialistes de la Thaïlande) ou commerciale (d'où la chasse aux contrefaçons). Mais les tentatives unilatérales pour faire respecter le territoire se heurtent souvent aux caractères diversifiés, multifonctionnels, fluides et inventifs des réseaux informels, d'autant que leur dimension transnationale induit l'implication de plusieurs gouvernements ${ }^{38}$. Dès lors, les espaces couverts par ces réseaux créent des contraintes pour les gouvernements qui veulent les contrôler ou les maîtriser. Ceux-ci doivent en effet s'engager dans des négociations impliquant tous ceux qui sont concernés, parce qu'ils sont soit au départ, soit à l'arrivée, soit en situation d'intermédiaires, de ces circuits d'échange. De nouvelles géographies de négociation et de coopération intergouvernementales se mettent ainsi en place entre des gouvernements qui, sans la présence de ces réseaux, n'auraient guère d'occasion ni de besoin d'aller au-delà de la simple courtoisie diplomatique. Les échanges de missions officielles, l'ouverture de lignes aériennes, la mise en place de véritables services publics chargés des pèlerinages, la conclusion de traités régulant la circulation des personnes et des biens, entre par exemple gouvernements d'Afrique orientale et de la péninsule arabe, doivent moins à des affinités idéologiques et même à des marchandages autour de livraisons de pétrole qu'à l'existence de flux continus et parallèles de population (main d'oeuvre, commerçants, entrepreneurs, pèlerins) et de biens divers (bijoux, épouses, hi-fi, alcools, dromadaires, dollars) entre les rives de l'océan indien occidental. L'informel transnational d'origine omani ou hadrami a contribué à la reconnaissance de l'importance du Yémen et du Sultanat d'Oman dans cet espace régional afro-arabe. Il y a de même un lien entre le statut international de Taïwan, Hong-Kong ou Singapour et l'activisme régional des Huaqiao, tandis qu'en cette fin de XXe siècle, les relations intergouvernementales en Europe centrale demeurent difficiles entre le gouvernement hongrois et ses voisins slovaques et roumains ou entre l'Allemagne et la République Tchèque en raison de la présence de minorités qui cherchent à préserver leur identité. Ainsi, les espaces des réseaux créent des situations nécessitant un traitement diplomatique et par conséquent la construction de nouveaux espaces de concertation intergouvernementale confirmant la relativité du sens du territoire étatique. Cette relativisation est encore plus nette, lorsque le traitement de l'informel transnational se traduit par des initiatives gouvernementales assimilables à des formes d'ingérence, contraires au principe de souveraineté territoriale, que ces initiatives soient prises avec 
ou sans l'agrément du gouvernement concerné. La délocalisation hors du territoire étatique d'actions policières ou militaires ponctuelles visant à démanteler les réseaux de trafiquants de drogue (des États-Unis vers l'Amérique andine par exemple), les mobilisations concertées d'organismes gouvernementaux et d'ONG pour intervenir de par le monde afin de protéger, nourrir ou soigner des populations (Afrique des GrandsLacs, corne de l'Afrique, et plus discrètement, sous continent indien ou Europe de l'est) et, finalement, les débats actuels entre théoriciens et entre chancelleries autour d'un droit ou d'un devoir d'ingérence montrent que les autorités publiques sont elles-mêmes amenées à repenser fondamentalement la souveraineté, notamment dans sa dimension territoriale, et les modalités de relations intergouvernementales du fait de la récurrence de manifestations de l'informel transnational, (pourquoi, le gouvernement français envoie-t-il des contingents au Rwanda?) et de la prise de conscience de l'existence d'enjeux résolument a-territoriaux ${ }^{39}$. Le sens traditionnel du territoire est bien déstabilisé publiquement par ses propres gardiens, qui jusqu'ici, n'hésitaient pas à le violenter, mais en recourant aux méthodes d'un informel transnational ancestral, officiellement clandestin, l'espionnage. Cette pratique traditionnelle rappelle que la prise en considération de l'informel transnational par les gouvernements n'est pas strictement liée à la vie internationale moderne. L'espionnage recourt en effet aux techniques de l'infiltration de réseaux préexistant susceptibles de servir de couverture. Mais ce n'est là qu'une modalité extrême des stratégies gouvernementales consistant à essayer de récupérer à leur profit les activités des réseaux, à défaut de pouvoir les éliminer. Confrontés à l'existence d'activités informelles fonctionnelles, dans le sens où elles répondent à des demandes sociales qui ne peuvent être satisfaites autrement, les autorités publiques peuvent hésiter à s'engager dans une politique répressive qui, bloquant le réseau, entraînerait le mécontentement non seulement des membres du réseau (perte de profits), mais aussi de la population (pénurie, besoin non satisfait). Ou si l'objectif répressif n'est pas atteint, le réseau parvenant à déjouer les manoeuvres des autorités, celles-ci se trouvent ridiculisées et discréditées. En toute hypothèse, un problème d'autorité doit être traité. Il peut être politiquement plus efficace non pas d'affronter l'informel, mais de tirer parti de sa fonctionnalité. Travailleurs, hommes d'affaires exilés, commerçants itinérants, réfugiés, touristes, pèlerins, professionnels "sans frontière", étudiants constituent des ressources potentielles pour un gouvernement désireux de nouer des alliances, de connaître ce qui se passe hors de ses frontières, ou tout simplement d'augmenter ses recettes. Les réseaux sont des vecteurs latents de présence à l'étranger et d'information qui ont l'avantage d'échapper à la rigidité formelle et aux contraintes imposées aux structures diplomatiques officielles. Ils peuvent ainsi servir de porteurs de messages ou de signaux discrets, au travers par exemple des pratiques sportives. La diplomatie du ping-pong de Nixon n'était qu'un exemple hautement médiatisé de cette récupération d'échanges transnationaux, y compris par une très grande puissance. En parallèle aux relations officielles, des gouvernements (aussi bien celui des pays d'origine que celui des pays d'accueil) peuvent trouver dans le secteur informel des informateurs discrets ou des émissaires noyés dans la population (pour ne pas parler à nouveau d'espions titulaires), susceptibles de fournir des renseignements précis et sûrs (plus précis et plus sûr que ceux de bien des diplomates de salon) aussi bien sur la situation politique que sur le contexte social, religieux ou économique de leur pays de résidence. Au-delà de la simple information, l'informel peut être le vecteur par lequel transitent, au mieux un transfert de connaissance (sorte de retour de «brain-drain » dont a pu bénéficier la 
Chine populaire, et qui inquiète une partie de l'opinion conservatrice américaine, en raison de la proportion croissante de diplômés d'origine étrangère dans les universités américaines), au pire un véritable pillage technologique ou industriel ou la subversion d'un système politique rival ${ }^{40}$. Mais quelle que soit l'importance de ces phénomènes de retour, l'informel transnational permet d'étendre de manière diffuse la zone d'extension d'un gouvernement soucieux de conforter sa position sur la scène internationale. Plus concrètement, les réseaux peuvent servir de points d'appui pour des initiatives diplomatiques valorisantes, comme la conduite d'une politique d'aide au développement, de transfert de technologie ou de relations diplomatiques privilégiées. Les gouvernements indiens et pakistanais rivalisent ainsi après de nombreux dirigeants du Tiers-monde en tirant parti de l'importance des populations indo-pakistanaises expatriées. Les gouvernements des pays arabo-musulmans jouent de même de l'existence de populations d'origine arabe et/ou islamisées pour obtenir le soutien au moins diplomatique de gouvernements africains dans les conflits qui les divisent (Libye-Egypte, Iran-Irak) et dans les négociations avec Israël. Ainsi, la répartition de l'aide des pays arabes producteurs de pétrole à l'Afrique était-elle directement proportionnelle à l'importance (réelle ou supposée) de la population musulmane africaine. La géographie des politiques gouvernementales est ici comme en Europe ou en Amérique du nord affectée par celle des réseaux transnationaux qui orientent le dépassement de l'espace territorial étatique, ce qui illustre l'importance que conserve le lien individuel - communautaire ${ }^{41}$. Ceci ne concerne pas seulement le positionnement sur la scène internationale, mais aussi la légitimité interne des gouvernants. Celle-ci peut être confortée par la valorisation de symboles de rayonnement international (la francophonie, la présence d'entreprises ou de ressortissants qui ont réussi...) mais aussi par le renforcement de ses capacités distributives, grâce aux revenus rapatriés par les travailleurs exilés ou aux investissements opérés par exemple en Chine continentale ou en Inde par des frères du bout du monde $\mathrm{e}^{42}$. Dans des proportions variables d'une période à une autre, d'un contexte à un autre, on peut alors dire que la prospérité relative d'une société étatique est parfois tributaire moins des revenus générés à partir de son territoire que des revenus drainés au travers de réseaux diffus éparpillés à travers le monde. S'il ne s'agit pas alors d'un "dépassement » à proprement parler du territoire, il s'agit d'une nouvelle preuve de la relativité de son sens, alors que se confirme la fonctionnalité de l'informel transnational au regard des intérêts des gouvernements. Il est donc non seulement impossible matériellement, mais maladroit politiquement, de se priver des ressources que cette transnationalité parallèle, clandestine ou illégale peut procurer. Le risque est d'aller au-delà d'une certaine idée de «l'intérêt de l'État» et d'essayer de profiter du caractère occulte ou diffus des activités du réseau et de sa propre position d'autorité publique pour se livrer à un chantage à des fins personnelles, que ce soit le maintien au pouvoir ou l'enrichissement privét ${ }^{43}$. La prospérité des réseaux informels dépend de la non intervention des autorités gouvernementales, celle-ci s'expliquant par l'ignorance (très importable) ou, comme nous venons de l'envisager, par la récupération des avantages qu'ils procurent. Elle peut aussi s'expliquer par une complicité personnalisée avec les membres du réseau pouvant aboutir à l'investissement de celui-ci par des dignitaires de l'appareil politique, administratif ou partisan. Dans un monde qui n'est pas naiff, l'abstention de celui qui a la garde de la frontière et du droit est rarement un geste de générosité pure. Il doit y trouver un intérêt, qui peut venir de sa propre appartenance personnelle à certains réseaux en 
toute honorabilité, mais le conduisant à être magnanime à l'égard des illégalités commises par ses frères ou disciples (islamisme militant de membres des forces de l'ordre égyptiennes ou algériennes). La faute professionnelle constitue le prix qu'il paie pour préserver ce qui lui parait être l'essentiel, à savoir l'appartenance au réseau. Mais en sens inverse, plus fréquemment sans doute, c'est l'auteur de l'illégalité qui doit rétribuer la non-intervention de l'autorité qui ne se contente pas de rétributions symboliques. Le prix est le résultat d'une négociation qui s'impose aux deux protagonistes car si l'activité informelle transnationale a besoin de l'abstention complice de celui qui matérialise la réalité de la frontière (le douanier, le policier), ce dernier sait que son gouvernement, ses compatriotes, lui-même ont besoin du réseau, notamment en ce qu'il pallie des carences de l'État ou qu'il apporte des satisfactions qui sont hors du champ étatique (en matière d'éthique par exemple). Lorsqu'il existe à l'évidence une disproportion considérable entre le chiffre d'affaires du réseau et le salaire du fonctionnaire qui est sur le terrain, celui-ci peut monnayer son inaction pour améliorer son ordinaire, et faire tout son possible pour que le flux rénumérateur ne se tarisse pas. Ainsi l'histoire populaire des sociétés transfrontalières (pyrénéenne par exemple, mais aussi bien andine ou himalayenne) est riche des récits de la complicité antagonique du garde-frontière et du contrebandier. La folklorisation de ces pratiques de survie, mais aussi ludiques et de convivialité, n'empêche pas qu'il s'agit de réalités toujours vivantes et lucratives, entretenant l'existence de communautés transfrontalières pour qui l'irruption de la frontière étatique n'a fait qu'ajouter une pièce supplémentaire à un jeu de société constamment renouvelé. Mais si la convivialité de l'espace local autorise une complicité sous le couvert d'apparences conflictuelles, la négociation corruptrice se durcit lorsqu'elle met en présence des acteurs importants, à la mesure des activités et surtout des profits du réseau. Les exemples ne manquent pas d'autorités publiques utilisant leur position institutionnelle pour accaparer, par les menaces et le chantage, tout ou partie des recettes et des avantages procurés par les activités informelles transnationales qu'ils savent ne pas pouvoir supprimer, ou qu'ils estiment plus intéressant de récupérer. La vie internationale est riche de gardiens de l'ordre étatique (hommes politiques, hauts fonctionnaires, chefs militaires, diplomates) menant une double vie grâce à "l'économie seconde», qu'il s'agisse de devises, de la commercialisation de la production nationale de café sur le marché international, de l'exportation illicite d'ivoire, de trophées ou d'or, ou tout simplement de placements à Zürich. La petite chronique des grandes manoeuvres de la corruption internationale basée sur des flux clandestins de biens ou de personnes s'enrichit chaque jour des façons de faire de dignitaires de toute origine, de toute orientation politique, agissant au mépris du territoire qui fonde leur légitimité. Ainsi, il n'est pas sûr que ceux qui sont les gardiens de l'État (et donc du territoire) respectent leur propre discours officiel valorisant le respect du territoire étatique. La banalisation de leur présence dans le partage des bénéfices retirés d'activités informelles transnationales (fussent-elles moralement acceptables), s'ajoutant à d'autres tendances lourdes de la vie internationale («mondialisation »), contribue à une dévalorisation généralisée de l'espace territorial étatique au point de conduire les gouvernements eux-mêmes à inscrire le problème de la corruption transnationale à l'ordre du jour d'organisme intergouvernementaux comme l'OCDE ${ }^{44}$. Conclusion Le territoire étatique n'est plus seulement contesté par la permanence des flux transnationaux informels et la prise en considération des enjeux environnementaux. Il est aussi contesté par l'évolution globale de la société, qui 
privilégie de plus en plus l'idée de mobilité (dans les études - Erasmus -, dans le travail, dans la conquête de marchés, dans le savoir ou le loisir) au détriment de la stabilité, de l'ancrage auquel est associée la représentation idéologique de l'État et que symbolise le «territoire». La mondialisation démode le territoire en général (communication électronique) et le territoire étatique en particulier, dont l'image est encore dégradée par les pratiques néo-patrimoniales, dont la notoriété publique vulgarise largement l'image dévergondée d'un territoire-objet lucratif au détriment de l'image sublimée du territoire valeur politique. Après avoir été avant tout le fait des minorités opprimées, de populations en quête de survie, de communautés marchandes, de prophètes, de truands ou d'autorités publiques avides de fortune, de prestige et de pouvoir, il n'est pas surprenant que l'informel transnational ne cesse de trouver de nouveaux adeptes et se banalise, touchant toutes les catégories sociales, toutes les activités, y compris, au sein même de l'institution étatique, parmi les collectivités locales. Le recours à l'informel transnational est moins le fruit d'une perversité morale ou politique (ce qu'il est parfois) qu'une réaction ingénieuse pour échapper aux entraves, aux blocages, aux défaillances engendrées non seulement par les déficiences de l'État sous développé, mais aussi par l'excessive bureaucratisation de l'État moderne. "Des millions de gens de par le monde vivent ainsi dans des micro-espaces à la fois localisés et sans frontières (...). Les microcosmes où ils vivent communiquent en permanence avec des ensembles beaucoup plus vastes, souvent plus réels que l'univers abstrait des spécialistes du développement qui sillonnent les airs mais restent enfermés dans les étroites limites de leur réalité artificielle...» ${ }^{45}$. Si le monde est bien entré dans une dialectique de mondialisation (sous la bannière du libéralisme) et de relocalisation (avec une proximité qui n'est pas nécessairement géographique), le territoire étatique n'en finira pas de perdre du sens, et l'informel transnational demeurera une formule d'avenir. Mais ceci ne signifiera pas automatiquement « la revanche de la société civile».

\section{NOTES}

1. « Le territoire s'analyse (...) comme un construit social, c'est à dire comme le résultat d'une tentative (...) d'affecter, d'influencer ou de régir des personnes, des phénomènes ou des relations en délimitant et en contrôlant une aire géographique » écrit B. Badie (suivant R.D. Sack) dans sa définition du Territoire qu'il développe dans le Dictionnaire de la Science politique et des Institutions politiques, Paris, A. Colin, Coll. Cursus, 1994, p. 266. Voir bien sûr du même B. Badie, La fin des territoires, Paris, Fayard, 1995, pp. 35-70. Du point de vue de la pensée politique, Cf. M. Korinman, M. Ronai, Les idéologies du territoire, in F. Châtelet (dir.). Histoire des idéologies - Savoir et pouvoir du XVIII au XX ${ }^{\circ}$ siècle, Paris, Fayard, 1978, pp. 229-257.

2. Dans la mesure où nous essayons de traiter des pratiques de la vie internationale, il est essentiel d'abandonner le vocabulaire personnifiant « l'État », commodité de langage qui occulte totalement les mécanismes réels du pouvoir. Elle se justifie dans une analyse juridique, mais dans une perspective sociologique, parler de « volonté des États ou de relations interétatiques » n'a pas de sens puisqu'il s'agit d'étudier des 
agissements d'acteurs concrets, versatiles, précaires sous les couverts du titre d'autorité publique et d'acteurs privés. Un bon éclairage sur les diverses facettes de la problématique est fourni par le $\mathrm{n}^{\circ} 117$ de la Revue Internationale des Sciences Sociales dirigé par C.F. Alger, « Interconnexions entre le local et le mondial », août 1988, pp. 359-453.

3. Cf. par exemple pour l'Afrique, J. Herbst, « Migrations, the Politics of Protest, and State Consolidation in Africa », African Affairs, 89, 1990, pp. 183-203 ; J.O. Igue, B. Soule, L'Etat-entrepôt au Bénin, Paris, Karthala, 1992 ; J. Mac Gaffey (ed.) The Real Economy of Zaïre, Londres J. Currey, 1991 ; L. Roitman, « The Politics of Informal Markets in SubSaharan Africa, JL of Modern ", African. Studies, 28 (4), 1990, pp. 671-696; Voir aussi Centre africain d'études monétaires - Associations des Banques centrales africaines, Le commerce frontalier en Afrique, 1984, sl ; «L'Afrique sans frontière », Politique africaine, $n^{\circ}$ 9, Mars 1983, pp. 3-83 ; M. Djama, « Dynamiques sociales en zone frontalière » (dans une communauté somali du Hawd), Paris, CNRS/EHESS, Recueils estafricains $\mathrm{n}^{\circ}$ 3, 1993 ; S. Ricca, La migration internationale en Afrique, Paris, L'Harmattan, 1990 ; Dossier « Le secret, c'est le réseau » (en Afrique), Croissance, n 386, oct. 1995, pp. 30-43, etc.

4. Selon une étude taïwanaise in C.I., $n^{\circ} 160,25.11 .1993$, les 55 millions de Chinois constituant la diaspora en Asie génèrent un produit national d'environ $500 \mathrm{Mds} \$$ et disposent de réserves en devises de 217,8 Mds \$ (contre 162,6 Mds pour le Japon et les États-Unis réunis) ; elle détiendrait 73\% du capital en Indonésie (hors secteur foncier), $61 \%$ du capital en actions en Malaisie, C.I., $n^{\circ} 257,05-11.10 .1995$. D'après une source japonaise, la diaspora philippine aurait rapatrié environ 10 Mds \$ (en 1993) dans un pays dont le PIB est estimé à 53,7 Mds \$, C.I. n 187, 2 08.06.1994. Le revenu annuel total des quelques dix millions d'Indiens expatriés serait d'environ $340 \mathrm{Mds} \$$, soit à peu près l'équivalent du PIB des Indiens de la métropole, C.I., $\left.n^{\circ} 254,13-19.07 .1995\right)$, etc.

5. J.F. Bayart a réunis les textes fondateurs de cette approche in J.F. Bayart, A. Mbembe, C. Toulabor (dir.), Le politique par le bas en Afrique noire, Paris, Karthala, 1992.

6. Nous avons esquissé la transposition in « Sur les modes populaires d'action diplomatique : affaires de famille et affaires d'Etat en Afrique orientale », Rev. Franç. Sc. Pol, n 36 (5), 1986, pp. 672-694. Voir aussi notre « A propos de l'étude des relations internationales... » in B. Badie, M. Pellet (dir.), Les relations internationales à l'épreuve de la science politique, Mélanges Marcel Merle, Paris, Economica, 1993, pp. 181-192, et "Les relations internationales entre tradition et post-modernité ? ", Le Trimestre du Monde, 3e Trim. 1994, pp. 49-72. Auparavant, quelques avancées sur cette piste avaient été faites par C.F. Alger, «Bridging the micro and macro in International Relations Research ", Alternatives, 1984-85, T X, pp. 319-344, et RISS, op. cit. Plus récemment, la thématique de la mondialisation par le bas « Globalization from below » a été exposée dans l'introduction de J. Brecher, J.B Childs et J. Cutler (eds), Global Visions. Beyond the New World Order, Boston, South End Press, 1993, ix-xxiv.

7. B. Badie, M.C. Smouts, Le Retournement du monde, Paris, Presses FNSP-Dalloz, 1993, p. 70.

8. B. Lautier, L'économie informelle dans le tiers monde, Paris, La Découverte, 1994, pp. 14-17.

9. Cf. par exemple J. Mac Gaffey, op. cit., pp. 12-15.

10. A. Colonomos, « Sociologie et science politique : les réseaux, théories et objets d'études », RFSP, 45 (1) 1995, pp. 165-178 ; A. Degenne, M. Forsé, Les réseaux sociaux, une analyse structurale en sociologie, Paris, A. Colin, 1994. Voir aussi Ph. Dujardin 
(dir.), Du groupe au réseau, Paris, CNRS, 1988 ; « Les liens sociaux invisibles », Thème de Sciences Humaines, hors Série, mai-juin 1994.

11. J. Lagroye, Sociologie politique, Paris, Presses FNSP/Dalloz, 1991, p. 198.

12. Cf. F. Constantin, C. Coulon, « La difficile décolonisation de la diplomatie africaine », in L'évolution récente du pouvoir en Afrique noire, Bordeaux, CEAN/IEP, 1978, pp. 219-236 ; F. Constantin, « Patronage et cardinalité », in D. Bach, A. Kirk Greene (dir.), Etats et sociétés en Afrique francophone, Paris, Economica, 1993, pp. 201-218; R. Lemarchand, Comparative Political Clientelism : Structure, Process and Optic, in S.N Eisenstadt, R. Lemarchand (eds), Political Clientelism, Patronage and Development, Beverly Hills, Sage, 1981, pp. 7-32.

13. D.C. Martin, « Le choix d'identité », RFSP, 42 (4), 1992, p. 588. Plus que de « lieu », on pourrait parler de « lien ».

14. B. Badie et M.C. Smouts rappellent ce positionnement des questions environnementales comme enjeu international inscrit dans le débat sur les biens communs, op. cit. pp. 216-235. Bien évidemment, cette prise en considération est intervenue bien avant la Conférence de Rio, avec les débats sur la " croissance zéro » (Halte à la croissance, Paris, Fayard, 1972), puis sur le « développement durable » (M. Jacobs, The Green Economy : Environment Sustainable Development and the Politics of the Future, Londres, Pluto Press, 1991, ou « Environnement, prévoir l'incertain », Thème de Projet, $n^{\circ} 226$, été 1991). A titre d'exemple, voir les problèmes de l'eau évoqués in Le Monde, 16.08.1995.

15. E. Fottorino, La piste blanche, L'Afrique sous l'emprise de la drogue, Paris, Balland, 1991 ; A. Labrousse, A. Wallon (dir.) La planète des drogues, Paris, Seuil, 1993 ; « The Drug Trade », dossier du Southerm African Economist, 5 (5), Sept. 1992, pp. 3-12. Le caractère très médiatique et très commercial du sujet suscite une abondante production écrite éclairant tous les aspects de ce genre de trafics.

16. J. Buob, Le Monde, 08.03.1995 ; D. Nora, Les conquérants du cybermonde, Paris, Calmann-Lévy, 1995. Une illustration très concrète des incidences de ce développement est donnée par le journaliste japonais H. Tamura sous le titre « L'Internet trouble Singapour. Le pouvoir se sent menacé par le développement du réseau », C.I., n 254 , 14-20.09.1995).

17. Voir par exemple les témoignages sur les réseaux indiens (reproduits in C.I., $\mathrm{n}^{\circ} 161$, 02.12.1993 et $245,13-19.07 .1995)$ ou chinois (ibid. 123, 12.03.1993; 160, 25.11.1993 ; $n^{\circ} 257,05.11 .10 .1995$; Le Monde, 31.07.1990). Notre article (RFSP, 36 (5) 1986) analyse certaines dimensions des réseaux indiens. Sur la diaspora chinoise, voir S. Boisseau du Rocher « L'État et le communautarisme chinois dans les pays de l'ASEAN », Cultures \& conflits, 15/16, 1994, pp. 127-163.

18. F. Constantin, «La transnationalité : de l'individu à l'État... », in M. Girard (dir.), Les individus dans la politique internationale, Paris, Economica, 1994, pp. 155-177.

19. Cf. les reportages sur certaines villes frontières finnoises comme Lappeenranta (C.I., $\mathrm{n}^{\circ} 245$, 29.06-05.07.1995) ou Vaalimaa (Le Monde, 02.11.1995), ou sur les pourtours de la Pologne (C.I., 231, 06-12.04.1995), le long du Rio Grande (C.I., n² 204, 06-12.10.1994 ; $n^{\circ} 243$, 29.06-05.07.1995 ; Le Monde, 17.12.1991, 13.01.1995 ; pour l'Afrique, voir par exemple. J. Igue, op. cit.

20. B. Lautier, op. cit., p. 35 ; J.M. Rodrigo, Le Sentier de l'audace : les organisations populaires à la conquête du Pérou, Paris, L'Harmattan, 1990.

21. G. L. Gardet, Les hommes de l'islam, Paris, Ed. Complexe, 1984. 
22. Les Chinois d'outre-mer seraient les principaux investisseurs en Chine continentale (10 Mds \$) ; mais les grandes familles expatriées privilégient les investissements dans la région où se trouve leur foyer d'origine (C.I., $\left.n^{\circ} 123,11.03 .93\right)$. De la même façon, nous avons pu noter que les investisseurs publics et privés séoudiens ou des émirats en Afrique orientale donnent la priorité aux projets portés par et concernant des populations musulmans.

23. Cf. J. Painter, Bolivia and Coca, a Study in Dependency, Boulder, Lyune Rienner, 1994 ; F.E. Thoumi, Political Economy and Illegal Drugs in Colombia, Boulder, Lynne Rienner, 1995 ; Voir par exemple C.I., n² 256, 29.09-04.10.1995, et sur les capacités de reconversion du réseau, C.I., n 263, 16-22.11.1995 .

24. Cité dans C.I, $n^{\circ} 223$, 09-15.02.1995.

25. O. Young, Arctic Politics ; Conflict and Cooperation in the Circumpolar North, Hanover, University Press of New England, 1992 ; PG Forest, R. Rodon, « Les activités internationales des autochtones du Canada ", Études internationales XXVL (1), 1995, pp. 35-58; O.S. Stokke, O. Tunander (eds), The Barents Region. Cooperation in Arctic Europe, Londres, Sage, 1994 (PRIO) ; C.I., n 124, 18.03.1993 ; Le Monde, 04-05.12.1994. 26. C.I., $n^{\circ} 230,30.03-05.04 .1995$.

27. Voir par exemple J.O. Igué, BG Soule, op. cit. Les VietKieu sont les vietnamiens en exil, C.I., nº 192, 7-12.07.1994.

28. Les scènes de la vie ordinaire décrites par P. Sansot, Les gens de peu, Paris, PUF, 1991, auraient pu être complétées par un tableau évoquant la transnationalité modeste au quotidien.

29. Les scènes de la vie ordinaire décrites par P. Sansot, Les gens de peu, Paris, PUF, 1991, auraient pu être complétées par un tableau évoquant la transnationalité modeste au quotidien.

30. « Internet, la démocratie en péril », Dossier de C.I., n 258, 12-18.10.1995.

31. Cf. pour l'Afrique australe le dossier établi sous le titre UTT (Unrecorded Transborder Trade), New name for an old game Southern African Economist, Dec. 1988/ Janv. 1989, pp. 3-13. Sur les développements du phénomène mafieux à partir de l'exURSS, cf. C.I., n¹40, 08.07.1993 (dossier) ; 201, 08-14.09.1994 (dossier) ; 227, 09-15.03.1995 ; 241, 15-21.06.1995 ; 244, 06-12.07.1995 ; Le Monde, 15.09.1995.

32. Outre, pour l'Afrique, S. Ricca, op. cit., voir S.S. Russel, K. Jacobsen, W. D. Stanley, International Migration and Development in Sub Saharan Africa, Washington, The World Bank, Discussion Paper 102 ; J. Conde, P. Diagne, NG. Ouaidou, South-North International Migrations. A Case Study, Paris, CAD/OCDE, 1986. Pour une approche plus large, R.T. Appleyard (ed.), International Migration Today, Paris, Unesco, 1988 ; B. Badie, C. Withold de Wenden (dir.), Le défi migratoire, Paris, Presses FNSP, 1994 ; D. Bigo, «Les États face aux flux transfrontaliers de personnes », Les Cahiers de la Sécurité Intérieure, $\mathrm{n}^{\circ}$ 19, 1er trim. 1995, pp. 115-125 ; P. Bernasconi, « La criminalité transfrontalière : sophistication financière et faiblesses judiciaires », Ibid, pp. 126-132. Sur les aspects pervers de ces migrations, illustrés par l'affaire Sarah Balabagan, cf. Le Monde, 11.10.1995.

33. Cf. Le Monde 26.04.1995. Voir par exemple les tarifs de la "Tête de serpent », filière d'émigration de main d'oeuvre chinoise continentale à destination de pays voisins in C.I. $\mathrm{n}^{\circ}$ 151, 23.09.1993, ou, dans l'espace de Schengen, « Les immigrants, nouvelles marchandises des mafias ", C.I., n² 236, 11-17.05.1995.

34. Sur la contrebande des produits alimentaires, cf. Rukanangira wa Nkera, B.G. Schoepf, « Unrecorded Trade in Southeast Shaba and across Zaïre's Southern Borders », 
in J. Mac Gaffey (ed.), op. cit., pp. 72-96 ; M.M. Mavambu ye Beda, The Trade in Food Crops, Manufactured Goods and Mineral Products in the Frontier Zone of Luozi, Lower Zaïre, Ibid., pp. 97-123.

35. Les pressions des autorités locales ont ainsi conduit à la mise en place d'un Conseil consultatif des autorités régionales et locales auprès de la Commission européenne en 1988. Cf. P. Blain, « The External Relations of Regions and Local Authorities ", in La répartition territoriale du pouvoir en Europe - II, Fribourg, Institut du Fédéralisme Fribourg, 1992, pp. 115-178; la problématique générale a déjà été explorée, notamment à partir des précédents canadiens, par I. D. Duchacek, D. Latouche, G. Stevenson (eds), Perforated Sovereignties and International Relations. Trans-Sovereign Contacts of Subnational Governments, New York, Greenwood Press, 1988. Voir aussi N. Loeb-Mayer, «Régions-Etats-Europe : des relations à l'échange » in P.H. Claeys, A.P. Frognier (dir.), L'échange politique, Bruxelles, Ed. de l'Université de Bruxelles, 1995, pp. 165-180. Le problème se pose aussi en Extrême-Orient, à partir de collectivités locales japonaises tournées vers la Russie, C.I., $n^{\circ} 256,29.09-04.10 .1995$ et d'autres enjeux internationaux, C.I., n² 262, 09-15.11.1995.

36. Sur le néo-patrimonialisme, cf. J.-F. Médard, « L'État néo-patrimonial en Afrique noire ", in J.-F. Médard (dir.), États d'Afrique noire, Paris, Karthala, 1991, pp. 343-353. 37. Nous avons donné une illustration des difficultés rencontrées sur ce terrain, y compris dans un système politique en apparence autoritaire, in F. Constantin, F. Le Guennec-Coppens, « Dubaï-Street, Zanzibar... », Politique africaine, 30 juin 1988, pp. 7-21

38. Voir par exemple les difficultés rencontrées par les autorités de police pour maîtriser « les inforoutes du crime », C.I., n²26, 02-08.03.1995, ou encore la poursuite des trafics (de pétrole en particulier) dans l'espace kurde malgré la fermeture de la frontière irakienne par l'armée américaine, C.I., n² 233, 20-26.04.1995).

39. Voir le dossier intitulé « Ingérence : vers un nouveau droit international », Le Débat, n 67, nov-déc. 1991, pp.4-39. L'un des exemples actuels les plus connus est l'intervention des Etats-unis en Colombie pour lutter contre les trafiquants de drogue. Voir une analyse critique in C.I. n 254, 14-20.09.1995.

40. L'actualité de l'année 1995 donne l'exemple du soutien du gouvernement pakistanais aux étudiants en théologie (taliban) en guerre contre le gouvernement afghan.

41. Les exemples a contrario de la pertinence de cette analyse sont donnés par l'échec de tentatives d'organisations régionales africaines qui, se réduisant à des saintes alliances de princes, sont dépourvues de sens pour les populations intégrées dans des réseaux de vie quotidienne ayant leurs propres axes. La nécessité pour une politique gouvernementale de disposer d'appuis dans le domaine de l'informel pour construire un espace d'influence durable est illustrée par les difficultés françaises à dépasser les limites du « pré-carré » africain post-colonial et francophone faute d'une présence sur le terrain suffisamment active (échec de la tentative de redéploiement de la politique africaine de la France entre 1977 et 1983 : parmi différentes causes, l'absence de réseaux « français » en Afrique anglophone rendait hasardeuse cette tentative d'ouverture).

42. Voir note 5 (supra) des exemples de la contribution des expatriés au développement économique et social de leur pays d'origine. A une échelle plus modeste, la relance économique à Zanzibar est considérablement aidée par les investisseurs omanis. 
43. Les gouvernements peuvent en effet tirer parti de la présence de ces expatriés (surtout s'ils sont puissants) pour en faire soit les financeurs de budget national ou du parti (financements d'origine arabe du parti gouvernemental kenyan) soit les boucs émissaires des malheurs du peuple (discours accusateurs, discriminations, expropriations, pogroms, expulsions, la politique d'Idi Amin Dada en Ouganda n'étant que la partie émergée d'un iceberg de pratiques permanentes à travers le monde du Nord au Sud).

44. Cf. G. Bridies, « Haro sur la corruption internationale », Le Monde, 05.07.1995. A titre anecdotique, voir par exemple « La contrebande sous contrôle d'Etat entre Roumanie et Serbie », C.I., n 257, 05-11.10.1995.

45. M. Rahnema, « Processus de pouvoir et de régéneration des micro-espaces », RISS, op. cit., p. 410.

\section{INDEX}

Mots-clés : économie politique, entrepreunariat, Mondialisation, réseaux transnationaux, territoire(s) et territorialité, drogue, contrebande et trafic 